\title{
Injection behaviour of fractured reservoirs at near wellbore and far field areas: a CFD study.
}

OLUYEMI, G.F., KANIN, L. and NAIR, A.

2014 


\title{
Injection Behaviour of Fractured Reservoirs at Near Wellbore and Far Field Areas - A CFD Study ${ }^{\dagger}$
}

\author{
Gbenga Folorunso Oluyemi*, Limniyakul Kanin, and Arwin Nair \\ School of Engineering, Robert Gordon University \\ Aberdeen, AB10 7GJ, UK \\ *E-mail: g.f.oluyemi@rgu.ac.uk
}

\begin{abstract}
Flow partitioning in fractured reservoirs especially during injection and production operations poses a great challenge to the optimisation of these important field operations. For example, flow partitioning into the fracture from the matrix may limit production from a well if the drilling and completion design does not take into consideration the position and intensity of the fracture networks. In the same vein, during chemical injection into a fractured reservoir for remedial flow assurance related purposes, the injected chemical may be forced into fracture zones with relatively low flow resistance depriving the target zones of the much needed dosage of chemical. Though several solution options are available to the operators to solve some of these optimisation problems if known at the planning and design stage of field development, however, there are very few options available to them to evaluate the flow behaviour of their fractured reservoirs at both near wellbore and far field areas of the reservoirs. In this study, we used CFD to investigate the injection behaviour of fractured reservoirs at near wellbore and far field areas of the reservoir in both vertical and horizontal directions under conditions of near equal and widely varying fracture and matrix permeabilities. The results highlight the twin effects of vertical flow distance from the matrix sites to the fracture face and fracture/matrix permeability on pressure distribution and flow partitioning during injection operation. In addition, the results demonstrate the application of CFD as a potential tool for preliminary evaluation and characterization of fractured reservoirs.
\end{abstract}

$$
* * *
$$

\section{Introduction}

Approximately $60 \%$ of the world's hydrocarbon reserves are stored in naturally fractured reservoirs [1], especially in Middle East and North America, with a recovery factor of a mere $20 \%$ or less [2], hence the need to enhance recovery from this type of reservoirs through concerted field

\footnotetext{
${ }^{\dagger}$ Received 31.10.2013
} 
optimisation efforts. The main barriers to recovery enhancement include complex geology resulting in complex fluid flow patterns.

Unlike conventional reservoirs with single porosity, naturally fractured reservoirs are generally divided into two zones, rock matrix providing hydrocarbon storage and high permeability, low porosity fractures providing pathways or barriers to the flow of fluid from the reservoir to the wellbore.

One defining fluid flow phenomenon in fractured reservoirs is flow partitioning between the matrix and the fracture spaces. The fluid divides between and flow through the two continua with the rate and amount of fluid travelling through each continuum varying over time depending on the prevailing flow conditions. The proportion of fluid flow in fracture and matrix domains depends largely on the matrix lithology, the hydraulic properties of the fracture and the matrix and the prevailing stress regime in the environment of the fractured reservoir rock. Hence flow partitioning in fractured reservoirs is complex and its understanding is still limited $[3,4]$.

Many practical challenges associated with naturally fractured reservoirs, especially tight matrix reservoirs, arise from their complexity and rather unpredictable nature of fluid flow through them. From reserves estimation perspective, there are great uncertainties as the fluid stored in the fractures may give an initial high production rate which then declines sharply $[5,6]$. From production perspective, excessive gas can be produced from the fractures. Similarly, from enhanced recovery perspective, injected fluids can channel through the fractures without sweeping the rock matrix.

This paper investigates the injection behaviour of fractured reservoirs under conditions of near equal and widely varying fracture and matrix permeabilities using Computational Fluid Dynamics.

\section{Problem Conceptualisation}

A square geometry $9.15 \mathrm{~cm} \times 9.15 \mathrm{~cm}$ was selected to represent the reservoir with a smooth channel having a width of $0.15 \mathrm{~cm}$ across the entire length of the domain representing an open fracture. A fluid distribution zone $1 \mathrm{~cm} \times 9.15 \mathrm{~cm}$ was created to distribute the flowing fluid evenly across the matrix and fracture. The schematic of the domain is shown in Fig. 1.

A single phase, laminar flow in a smooth open fracture simulation was carried out in this study. Two-dimensional (2D) simulation was chosen due to its simplicity and reduced calculation time. The simulations were based on the following underlying assumptions:

1. Constant fluid density: incompressible fluid assumption is valid under normal subsurface conditions.

2. Steady-state flow: since the solutions are dependent on space and independent of time, the transient effect is not taken into consideration. In addition, steady-state flow simulation gives better understanding than transient flow simulation [1].

3. The reservoir rock has matrix permeability ranging between 0.1 and $30 \mathrm{mD}$; and fracture permeability ranging between $2 \cdot 10^{3}$ and $2 \cdot 10^{8} \mathrm{mD}$.

\section{CFD Numerical Modelling Set Up}

2.1. Geometry and meshing. Design Modeller in ANSYS Workbench was used to create the geometry model. The $9.15 \mathrm{~cm} \times 9.15 \mathrm{~cm}$ fractured reservoir domain was divided into 3 zones - 


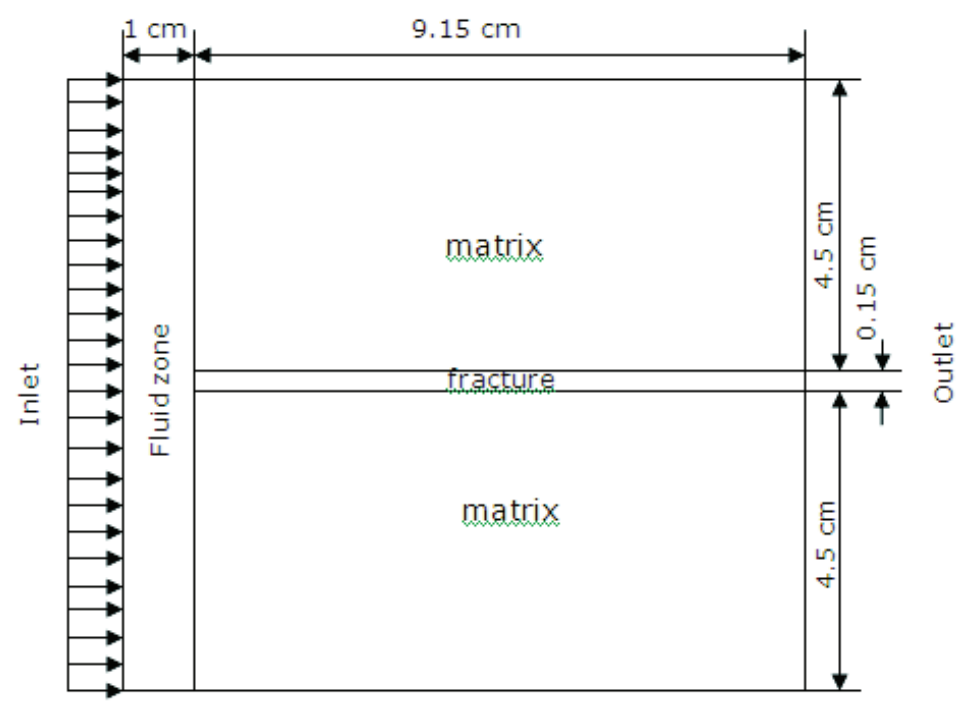

Fig. 1. Schematic design of the fractured rock geometry.

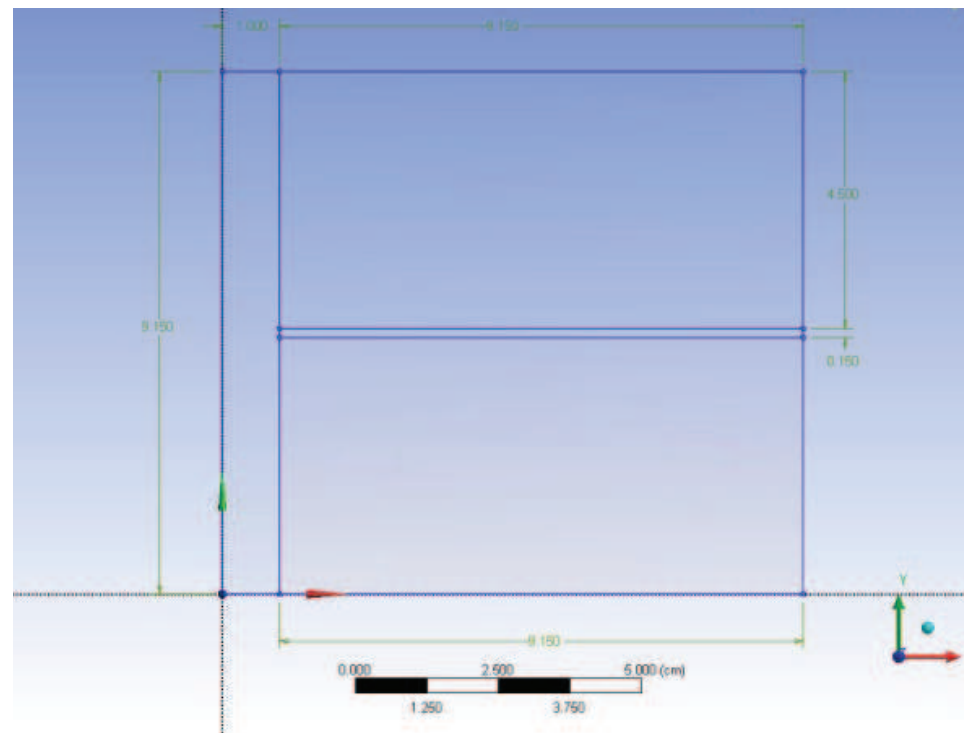

Fig. 2. Final geometry model. 
upper matrix zone, middle fracture zone and lower matrix zone. Another zone of $1 \mathrm{~cm} \times 9.15 \mathrm{~cm}$ was created to extend across the fractured reservoir face; the purpose of this zone being to help distribute the injection fluid evenly across the matrix and fracture. The final geometry model is presented in Fig. 2.

The geometry model was then imported into Fluent Meshing scheme. 37149 fine and uniform rectangular grids and 37536 nodes were generated; Fig. 3 shows the meshing refinement process.

The inlet and outlet boundaries were defined at the left hand and right hand sides of the geometry respectively to represent a flow direction moving from left to right. The two remaining edges were applied as a symmetry plane. A wall was defined at the entrance of the open fracture in order to see the flow interaction between the matrix and the fracture (Fig. 4).

2.2. Flow physics and boundary condition. As previously highlighted, the applied flow physics for this study was steady state, viscous laminar model. The flowing fluid was defined as oil with the input properties being fluid density, viscosity, matrix porosity and permeability, and fracture porosity and permeability.

The solver process was set up by employing an implicit, double-precision, two dimensions pressure based solver. Solution methods comprised of simple scheme for pressure-velocity coupling, a second-order upwind scheme for momentum discretization and a standard scheme for solving pressure. The solution convergence criteria consisted in the residual values of continuity, $x$-velocity and $y$-velocity solutions that were less than $10^{-6}$. The velocity inlet was used for initializing solution prior to the input of the maximum iteration number, which was 1000 . The calculation continued until the solutions converged or reached the specified number of iterations (Fig. 5)

2.3. Simulation. The simulations were designed to investigate the injection behaviour of tightmatrix fractured reservoir formation were run for both high and low matrix-fracture permeability ratios. The high matrix-fracture permeability ratio simulation was run using matrix permeability and porosity of $0.1 \mathrm{mD}$ and $40 \%$ respectively whilst fracture permeability and porosity of $2 \cdot 10^{8} \mathrm{mD}$ and $0.1 \%$ respectively were used, giving a high matrix-fracture permeability ratio of $1: 2000000000$. The inlet velocity was defined as $1 \mathrm{~m} / \mathrm{s}$ of oil $\left(\rho=850 \mathrm{~kg} / \mathrm{m}^{3}\right.$ and $\left.\mu=0.7 \mathrm{cP}\right)$.

For the low matrix-fracture permeability ratio simulation, matrix permeability and porosity of $20 \mathrm{mD}$ and $40 \%$ respectively were used whilst fracture permeability and porosity of $200 \mathrm{mD}$ and $0.1 \%$ respectively were used; giving a low matrix-fracture permeability of $1: 10$. The same fluid properties used for the high matrix-fracture permeability ratio simulation were also used for this simulation.

\section{Results and Discussion}

The results of the CFD simulation were analysed using $x-y$, contour and vector plots of the volume fractions. $x-y$ plots qualitatively show pressure and velocity variations along the reservoir domain, such as along or across the fracture whilst contour plots show the pressure and velocity distribution and magnitude along the domain. Vector plots provide clear direction of the flow through the fractured reservoir.

3.1. Injection behaviour of fractured reservoirs. Fig. 6 presents the pressure contours showing pressure distribution in the fractured domain during injection for the high matrix-fracture permeability simulation case. It shows substantial non-linearity in the distribution of pressure across the matrix and fracture domains, an indication of pronounced flow partitioning between the matrix and the fracture. Fig. 7 presents the pressure gradient across the matrix and fracture domains. 

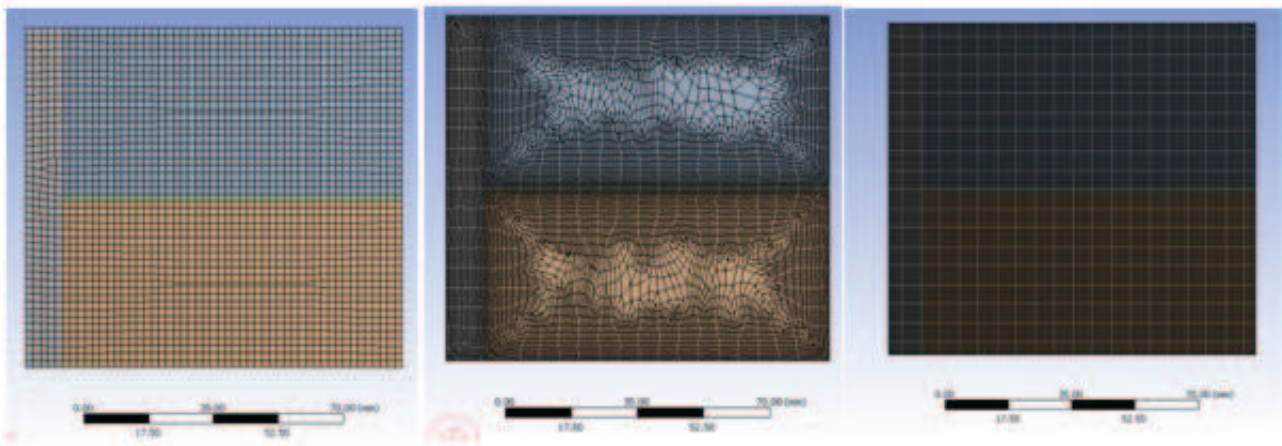

Fig. 3. Grid refinement process, default grid (left), refinement by edge sizing (middle) and mapped face meshing (right).

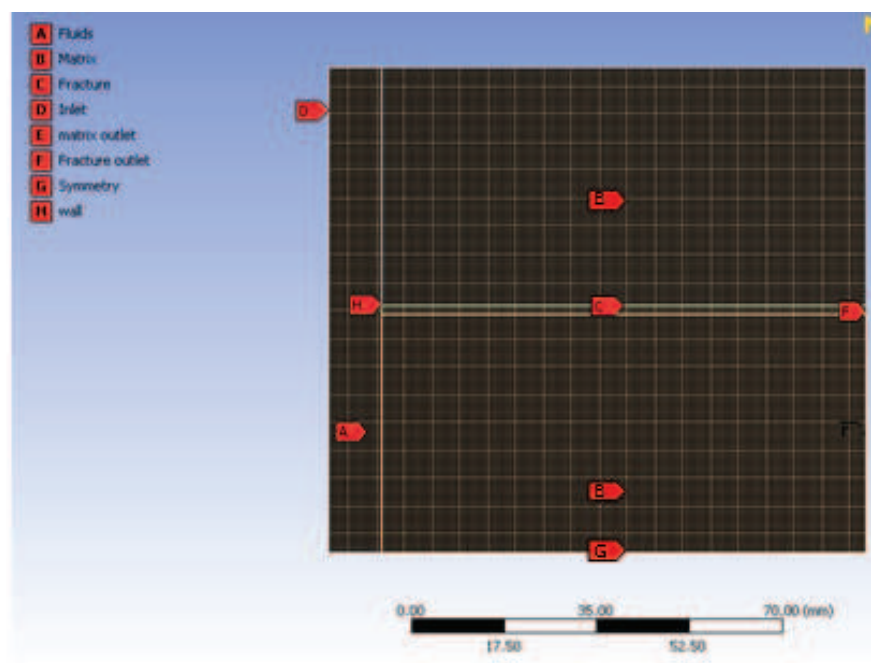

Fig. 4. Boundaries, matrix and fracture zones definition in meshing process.

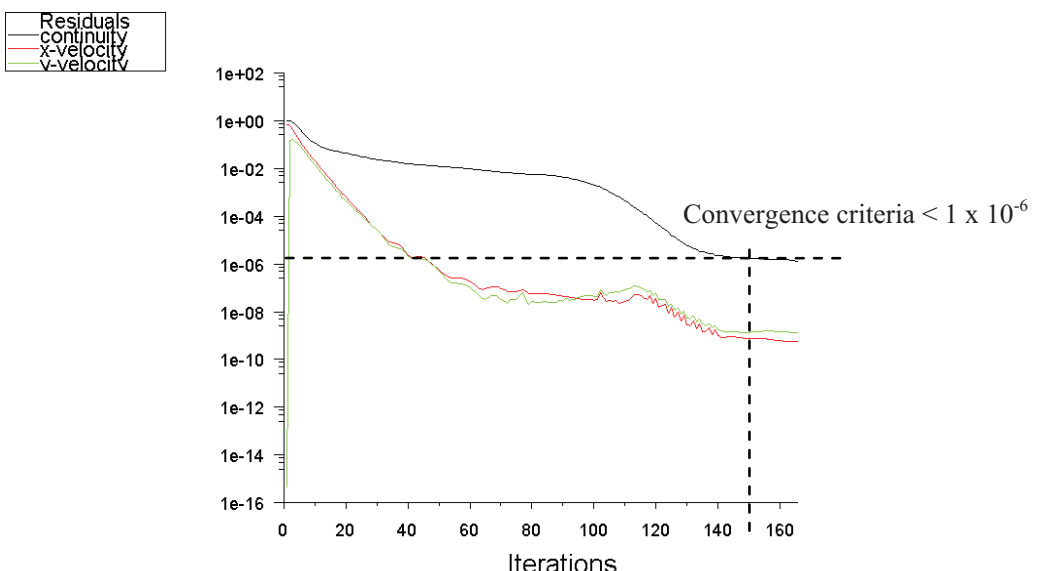

Fig. 5. Residual value of continuity, $x$-velocity and $y$-velocity; the calculation stopped after all 3 residuals were less than the convergence criteria at the 170-th iteration. 


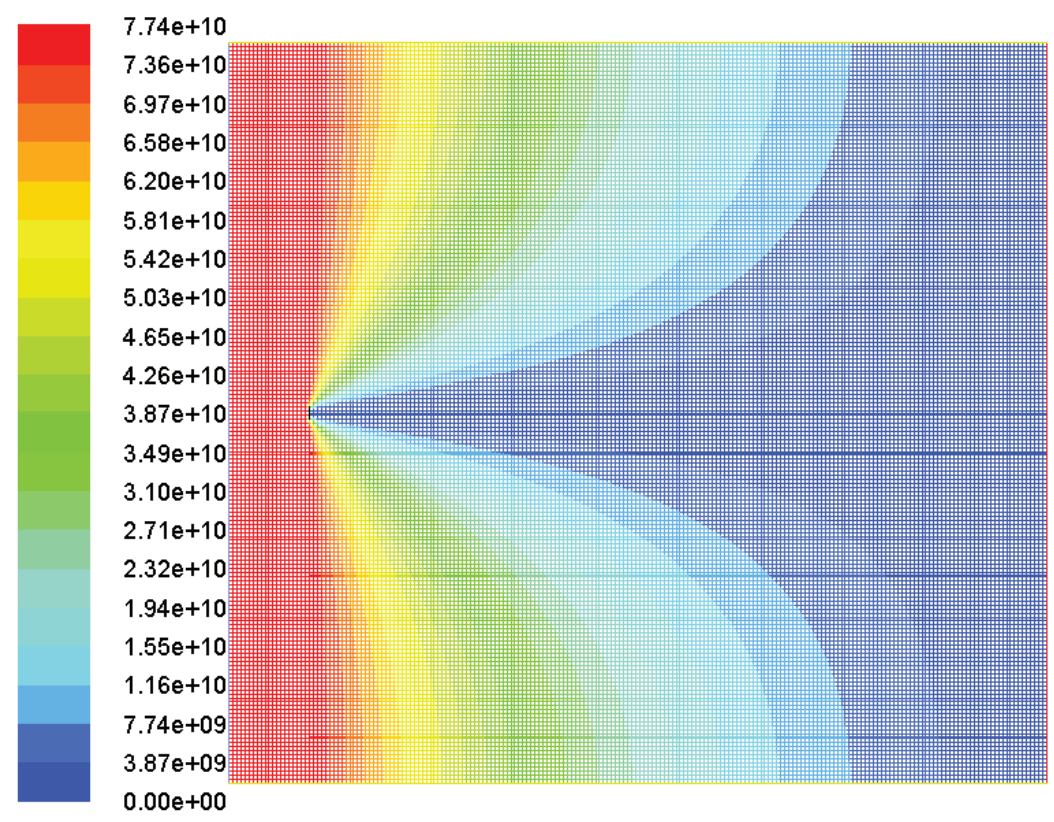

Fig. 6. Pressure countours of the entire fractured domain for the high matrix-fracture permeability simulation case with low pressure in the fracture and high pressure in the matrix away from the fracture.

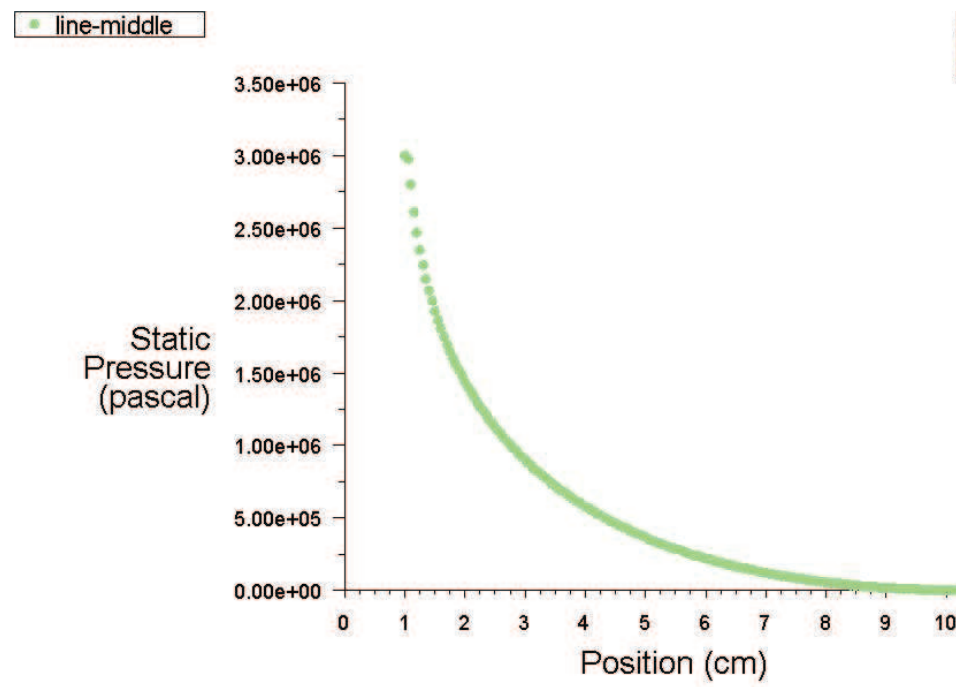

Fig. 7. The non-linearity of the pressure gradient across the entire fractured domain for the high matrix-fracture permeability simulation case.

Fig. 8 presents the pressure contours showing pressure distribution in the fractured domain during injection for the low matrix-fracture permeability simulation case. The figure shows uniform pressure distribution and linear pressure drops along the direction of the flow (Fig. 9). The fluid tends to flow uniformly across both the matrix and the fracture (Fig. 10) showing no disproportionate flow partitioning between them. 


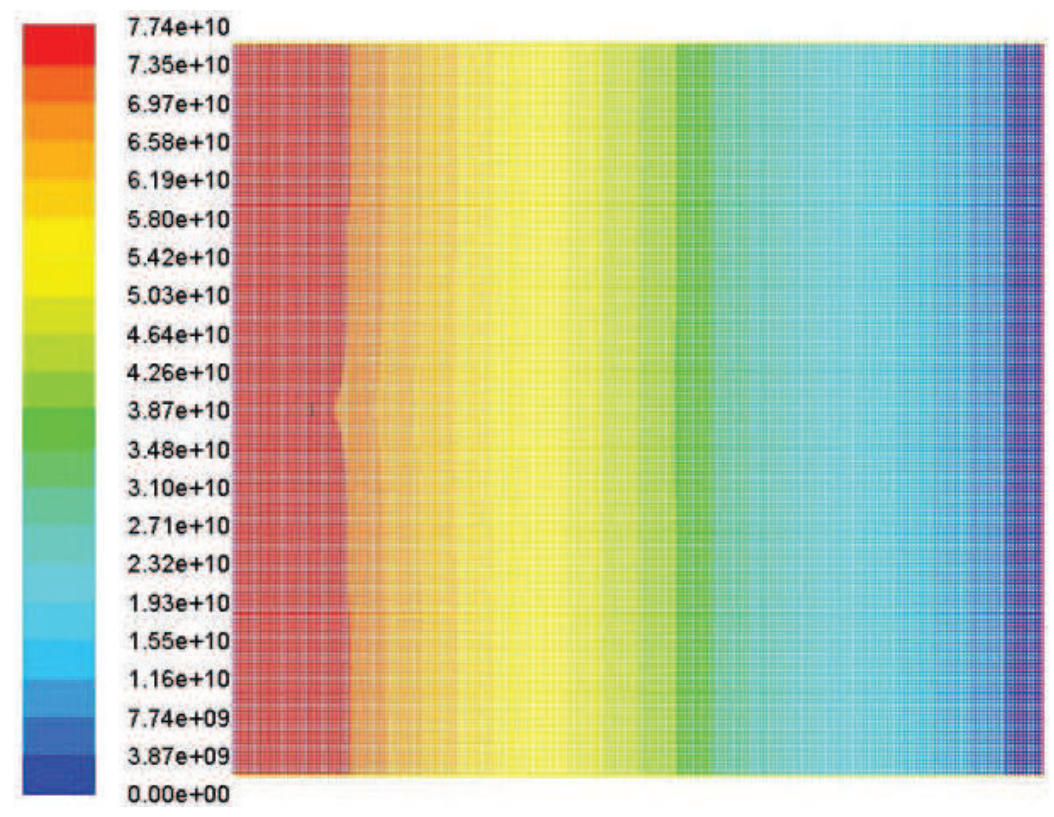

Fig. 8. Pressure countours of the entire fractured domain for the low matrix-fracture permeability simulation case with uniform pressure distribution across both matrix and fracture.

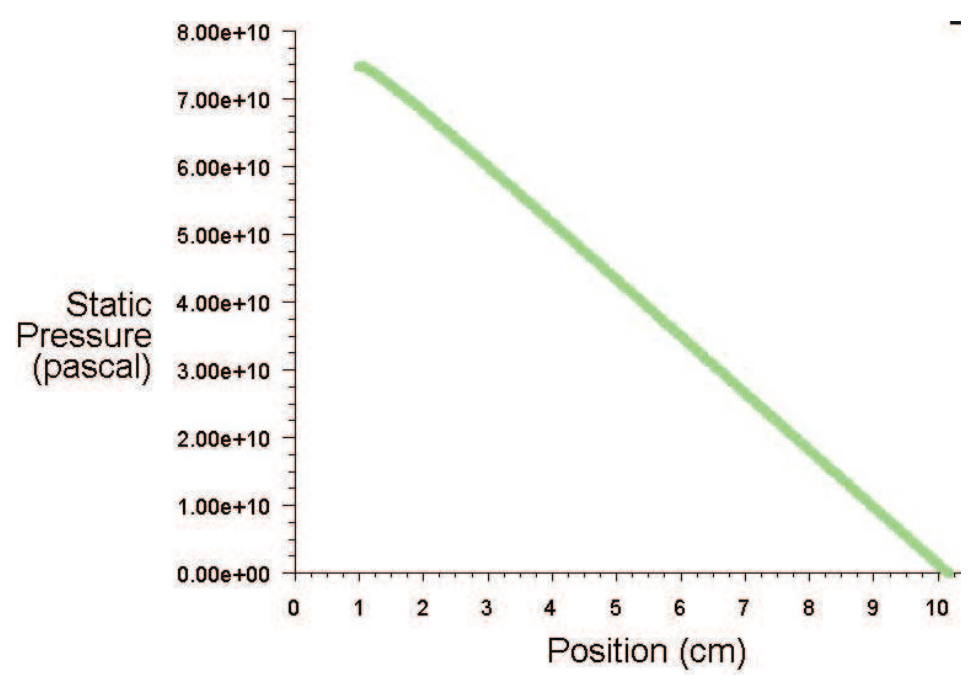

Fig. 9. The linear pressure gradient across the entire fractured domain for the low matrix-fracture permeability simulation case.

For the high matrix-fracture permeability simulation case, the parabolic velocity magnitude profile in the fracture observed quantitatively from the $x-y$ plot and in velocity vector form is presented in Figs. 11 and 12 respectively. The highest velocity magnitude of $8.9 \mathrm{~m} / \mathrm{s}$ at the centre of the fracture and the near zero velocity at the interface between the fracture and the matrix is evidence that most of the fluid at the near wellbore area of the fractured reservoir channels through the fracture space during flow. Furthermore, it is an indication of the magnitude of flow partitioning 

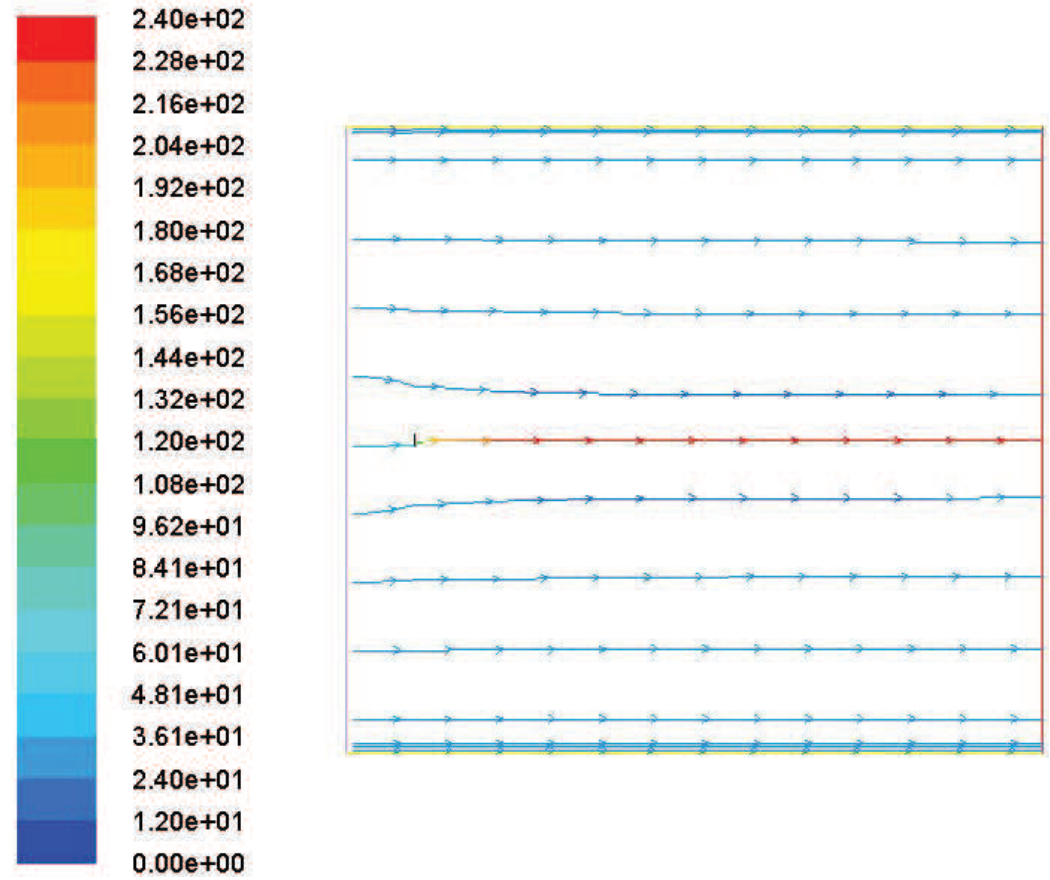

Fig. 10. The path lines showing no preferential flow through the fracture for the low matrix-fracture permeability simulation case.

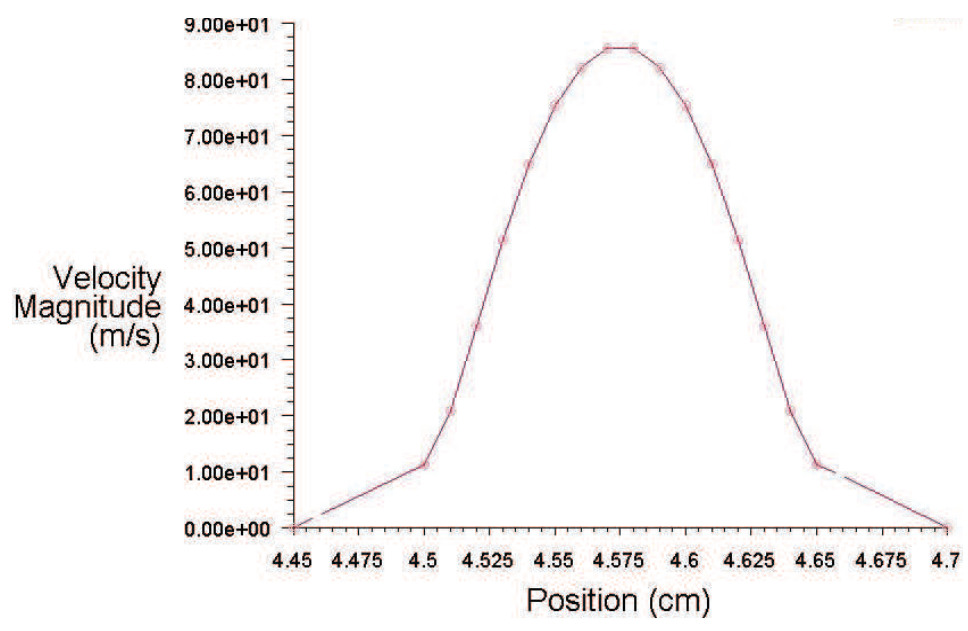

Fig. 11. $X-Y$ plot showing the parabolic velocity magnitude profile of the flow in the fracture for the high matrix-fracture permeability simulation case.

occuring within the fractured reservoirs.

3.2. Effect of vertical distance of inflow on flow partitioning. The flow partitioning pattern was observed by using a pathline graphic as shown in Fig. 13. Twelve different points were defined at the fractured geometry inlet on both sides at some predetermined distances from the fracture lateral position, which was used as a reference point. These points were $\pm 4.425, \pm 4.375, \pm 3.975$, $\pm 2.975, \pm 1.975$, and $\pm 0.975 \mathrm{~cm}$, and they represented the starting points of the flow pathlines. At 


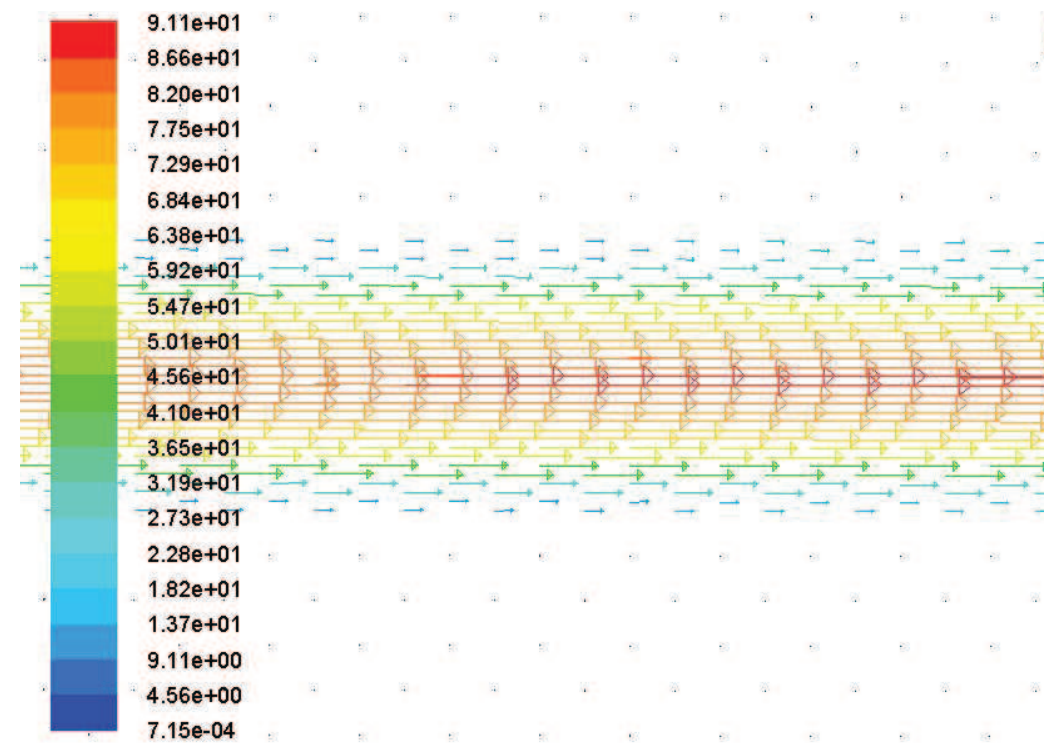

Fig. 12. Close-up velocity vector showing parabolic velocity magnitude profile of the flow in the fracture for the high matrix-fracture permeability simulation case.

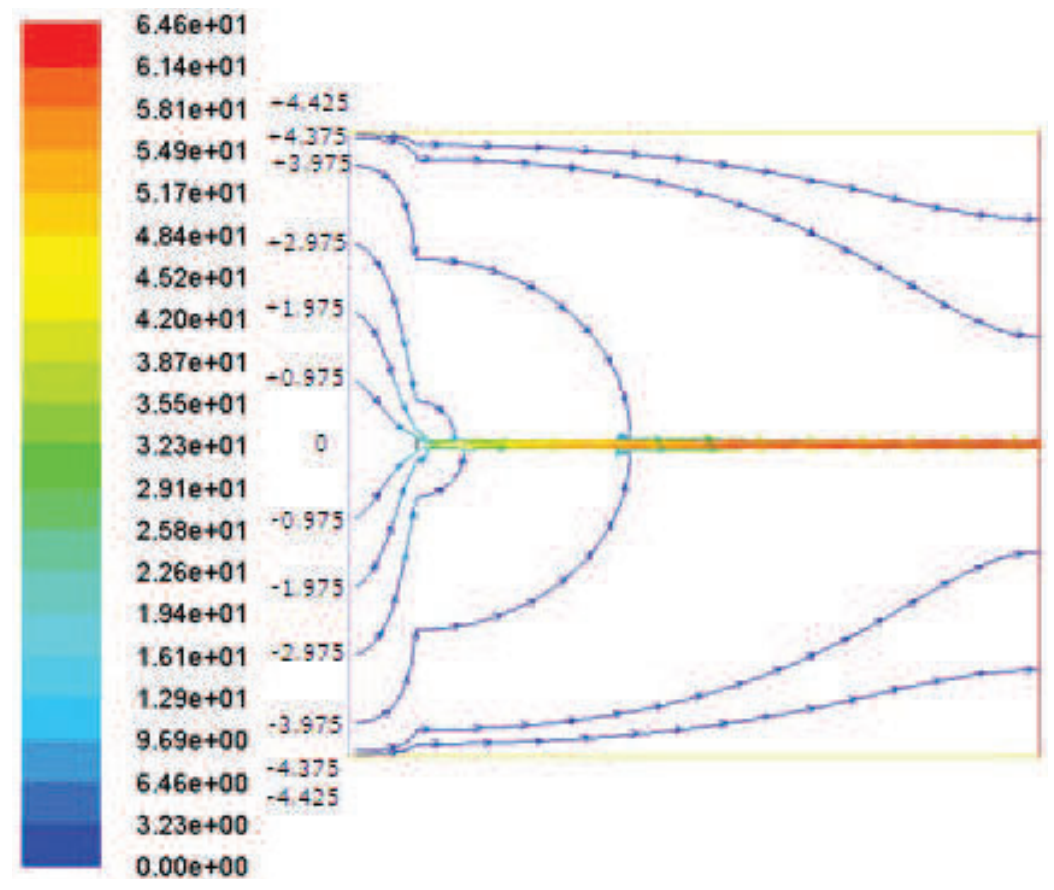

Fig. 13. Pathline graphic showing the behaviour of fracture reservoir during injection for the high matrix-fracture permeability simulation case. 
the points located between \pm 0.975 and $\pm 3.975 \mathrm{~cm}$ from the reference point (fracture lateral position), most of the fluid tends to flow slowly into the matrix, travelling a short distance before being diverted to the fracture due to its relatively high permeability. The horizontal distance travelled by the fluid within the matrix before being diverted to the fracture appears to be a function of the vertical distance of the flow entry point into the fractured rock from the lateral position of the fracture. However, at the points located \pm 4.425 and $\pm 4.375 \mathrm{~cm}$ away from this position, the fluid tends to flow through the matrix without being diverted into the fracture space at any instance during the flow. The plausible explanation for this behaviour is that the vertical distance of the fluid entry point must have overcome the influence of high permeability of the fracture. Fig. 14 shows the close up velocity vectors of the fluid flow from the fluid zone into the fracture.

\section{Conclusion}

Injection behaviour of fractured reservoirs has been simulated for both low and high matrixfracture permeability ratios. The injection behaviour of fractured reservoirs, when the matrixfracture permeability ratio is high, is such that the majority of the fluid flows through the fracture at the near wellbore area very close to the fracture lateral position. However, at some vertical distances from the fracture lateral position, most of the fluid flowing through the matrix tends to partition and flow into the fracture space as the flow progresses farther into the reservoir. The degree of partitioning and the amount of flow from the matrix to the fracture space appear to correlate directly with the vertical flow distance from the fracture lateral position and the horizontal flow distance from the near wellbore area. However, at certain vertical and horizontal flow distances, the partitioning of flow ceases and majority of the fluid flows through the matrix. Contrastingly, in the low matrixfracture permeability case the fluid flow is evenly distributed between the fracture and the matrix space showing no preferential flow through either the matrix or fracture space.

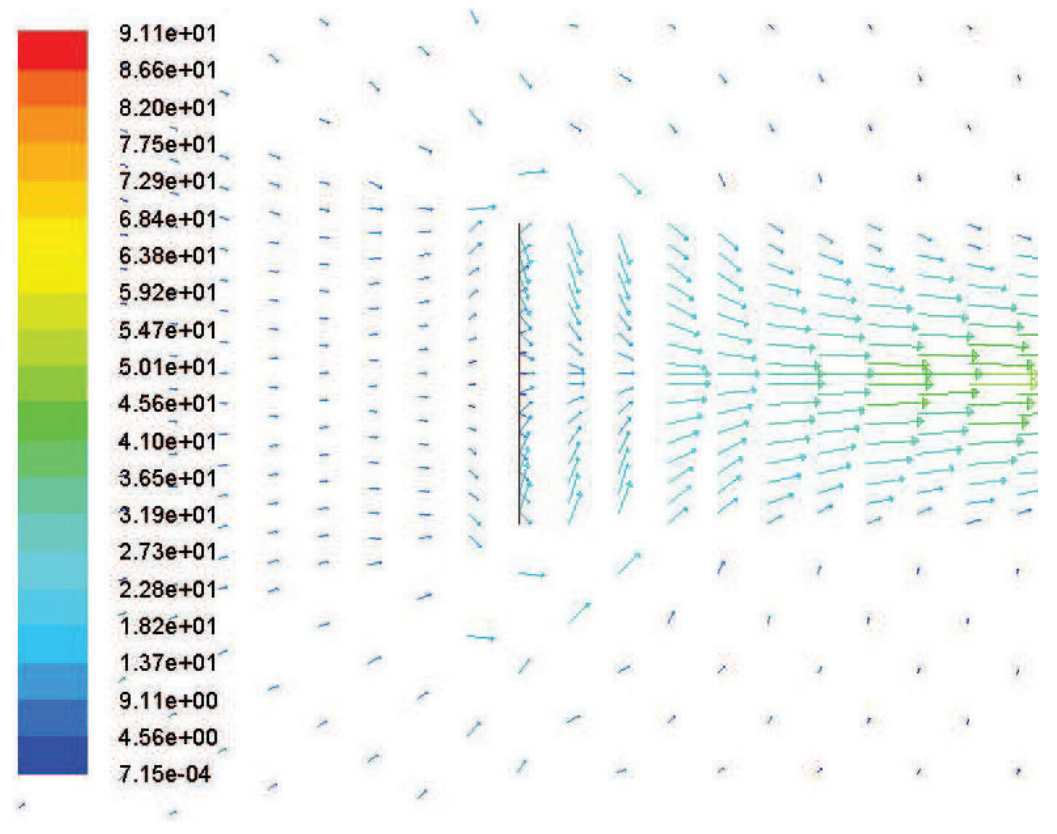

Fig. 14. Close-up picture of velocity vectors showing the fluid flow in the fracture for the high matrix-fracture permeability simulation case. 
In summary, the injection behaviour of fractured reservoirs is influenced by two important variables; these are the relative magnitude of matrix and fracture permeability and the vertical and horizontal distances of flow from the fracture space.

\section{REFERENCES}

1. Sarkar, S., Toksoz, N. and Burns, D. R., Fluid Flow Modelling in Fractures, Cambridge, MA, MTI, 2004 [http://www-eaps.mit.edu/erl/research/report1/pdf2004/sarkar.pdf, access. 10 July 2011].

2. Ozkaya, S. I. and Minton, K. R., Flow Potential of Fracture Corridors and Large Conductive Fractures in a Clastic Reservoir, Oman, In: Fractured Reservoirs, Lonergan, The Geological Society, London, Spec. Pub. 270, 2007, pp. 245-264.

3. Oluyemi, G. F. and Ola, O., Mathematical Modelling of the Effects of In-Situ Stress Regime on Fracture-Matrix Flow Partitioning in Fractured Reservoirs, In: 34-th Ann. SPE Int. Conf. Exhibit., Tinapa-Calabar, Nigeria, 2010.

4. Mathaii, S. K. and Belayneh, M., Fluid Flow Partitioning Between Fractures and a Permeable Rock Matrix, Geophys. Res. Lett., 2004, 31, No. 1, DOI: 10.1029/2003GL019027.

5. Aguilera, R., Naturally Fractured Reservoirs, PennWell Publ. Co., Tulsa, 1995.

6. Aguilera, R., The Uncertainty of Evaluating Original Oil-In-Place in Naturally Fractured Reservoirs, In: SPWLA 19th. Ann. Symp. Trans., Soc. Petrophys. Well Log Analysts, 1978, pp. A1A17. 